\title{
Numerical simulation of rarefied gas flows with specified heat flux boundary conditions
}

\author{
Jianping Meng1, Yonghao Zhang ${ }^{1, *}$, Jason M. Reese ${ }^{2}$ \\ 1 James Weir Fluids Laboratory, Department of Mechanical \& Aerospace Engineer- \\ ing, University of Strathclyde, Glasgow, G1 1XJ, United Kingdom \\ ${ }^{2}$ School of Engineering, University of Edinburgh, Edinburgh, EH9 3JT, United King- \\ dom
}

\begin{abstract}
We investigate unidirectional rarefied flows confined between two infinite parallel plates with specified heat flux boundary conditions. Both Couette and forcedriven flows are considered. The flow behaviors are analyzed numerically by solving the Shakhov model of the Boltzmann equation. We find that the existence of an zero heat flux wall can significantly influence the flow behavior, including the velocity slip and temperature jump at the wall, especially for high-speed flows. The predicted bimodal-like temperature profile for force-driven flows cannot even be qualitatively captured by the Navier-Stokes-Fourier equations.
\end{abstract}

AMS subject classifications: 76P05, 82B40

Key words: specified heat flux boundary, rarefied gas flow, $\mathrm{S}$ model, discrete velocity method

\section{Introduction}

In a broad range of engineering applications, the characteristic spatial scale is comparable to the mean free path of the working gas. These application range from high altitude and high speed space vehicles [1] to Micro-Electro-Mechanical systems (MEMS) [2]. In such conditions, typically, the conventional Navier-Stokes-Fourier (NSF) equations are invalid as rarefaction effects become substantial. Kinetic methods such as direct simulation Mote Carlo (DSMC) [3] or direct solution of the Boltzmann equation [4] become neccessary. However, the computational costs of these methods are high. In particular, the signal/noise ratio is typically low for flows in MEMS making the computational cost significant for DSMC. Various techniques have been proposed to improve computational efficiency, e.g. [5-8].

Thermal management is often of great importance in many applications, e.g., reentry vehicle thermal protection systems in which insulation techniques are used. To

\footnotetext{
*Corresponding author. Email address: jianping.meng@strath.ac.uk (J. P. Meng), yonghao.zhang@strath.ac.uk (Y. H. Zhang), jason.reese@ed.ac.uk (J. M. Reese)
} 
describe such systems, it is important to employ heat flux boundary conditions so that the heat exchange between the system and the surroundings can be controlled [9-18]. Particularly, in a well insulated surface, the adiabatic boundary condition may become appropriate for flow modeling. For this purpose, a immediate way is to set the accommodation coefficient in the commonly used Maxwell-type boundary to zero, i.e., full specular reflection, see the discussion in [11] and an example in [15]. Naturally, this kind of implementation is typically corresponding to a smooth surface. However, for rough surfaces, the heat exchange control is also possible. Therefore, it is necessary to discuss the specified heat flux boundary condition under a full diffusereflection condition. A few implementations have been explicitly discussed for the DSMC method [9, 10]. However, in terms of kinetic model equations, there is lack of such discussion and understanding of flow features even for simple flows.

In this paper, we report numerical investigations of rarefied gas flows confined between two parallel infinite plates. We solve the Shakhov model (S model) of the Boltzmann equation [19,20]. While one plate is set to be with specified heat flux (mainly zero flux), the other one has a fixed temperature. Here we suppose that the zero heat flux boundary can closely resemble the adiabatic boundary condition ${ }^{\dagger}$. The numerical method we use is based on the deterministic discrete velocity method (see [21] and reference therein). Both a Couette flow and a force-driven Poiseuille flow are examined.

\section{Formulation of problems}

\subsection{Specification}

The monatomic gas is confined between two parallel infinite plates located at $y=0$ and $y=L$. The top plate $(y=L)$ has a fixed temperature, $T_{0}$, and the bottom one $(y=0)$ has a zero heat flux. In the Couette flow, the two plates move oppositely with the same speed $U_{w}$. For the Poiseuille flow, the gas is subject to a uniform external force in the $x$ direction, i.e. in the direction parallel to the plates.

\subsection{Model}

In order to capture rarefaction effects, we solve the $S$ model of the Boltzmann equation which is given as:

$$
\frac{\partial f}{\partial t}+c \cdot \frac{\partial f}{\partial r}+g \cdot \frac{\partial f}{\partial c}=\frac{1}{\tau}\left(f_{S}-f\right),
$$

where $f(\boldsymbol{r}, \boldsymbol{c}, t)$ is the single molecular velocity distribution function, which describes the number (or portion) of molecules in the volume $d \boldsymbol{r}$ centered at the position $r=(x, y, z)$ with velocities within $d c$ around the velocity $c=\left(c_{x}, c_{y}, c_{z}\right)$ at time $t$. Based on this distribution

\footnotetext{
${ }^{\dagger}$ Under rarefied conditions, it becomes rather difficult to precisely define the adiabatic boundary condition, in particular for the moving wall. There may be different kinds of definitions, e.g. |22]. However, we can always use a specified heat flux boundary condition (may not be zero) to describe it phenomenologically.
} 
function, macroscopic quantities such as gas density $\rho$, velocity $\boldsymbol{u}$, temperature $T$, stress tensor $\boldsymbol{P}$ and heat flux $\boldsymbol{q}$ can be obtained from its moments i.e.,

$$
\rho\left[1, u_{i}, 3 R T, P_{i j}, 2 q_{i}\right]=\int\left[1, c_{i}, C_{i} C_{i}, C_{i} C_{j}, C_{i} C_{j} C_{j}\right] f d c,
$$

where $\boldsymbol{C}=\boldsymbol{c}-\boldsymbol{u}$ is the peculiar molecular velocity and $R$ is the gas constant. $\tau$ is the relaxation time with the form of $\tau=p / \mu$ where $p$ is the pressure and $\mu$ the viscosity. The body force $g=\left(g_{x}, g_{y}, g_{z}\right)$ is assumed to be independent of the molecular velocity. The "equilibrium" function $f_{S}$ is proposed to be

$$
f_{S}=f_{e q}\left[1+\frac{1-\operatorname{Pr}}{5} \frac{2 q_{i} C_{i}}{p R T}\left(\frac{C^{2}}{2 R T}-\frac{5}{2}\right)\right],
$$

where $f_{e q}$ is the Maxwellian distribution

$$
f_{e q}=\rho\left(\frac{1}{2 \pi R T}\right)^{3 / 2} \exp \left[-\frac{C^{2}}{2 R T}\right]
$$

and Pr is the Prandtl number.

It is convenient to introduce the following dimensionless quantities,

$$
\begin{aligned}
& \hat{x}_{k}=\frac{x_{k}}{L}, \quad \hat{u}_{k}=\frac{u_{k}}{\sqrt{R T_{0}}}, \quad \hat{t}=\frac{\sqrt{R T_{0}} t}{L}, \quad \hat{g}_{k}=\frac{L g_{k}}{R T_{0}}, \quad \hat{c}_{k}=\frac{c_{k}}{\sqrt{R T_{0}}}, \quad \hat{T}=\frac{T}{T_{0}}, \\
& \hat{f}=\frac{f\left(R T_{0}\right)^{3 / 2}}{\rho_{0}}, \quad \hat{\rho}=\frac{\rho}{\rho_{0}}, \quad \hat{p}=\frac{p}{p_{0}}, \quad \hat{\mu}=\frac{\mu}{\mu_{0}}, \quad \hat{q}_{i}=\frac{q_{i}}{p_{0} \sqrt{R T_{0}}}, \quad \hat{P_{i j}}=\frac{P_{i j}}{p_{0}} .
\end{aligned}
$$

Here, we use the subscript 0 to denote the reference quantities. For the present problems, most of them are corresponding to quantities at the top plate, except that the density $\rho_{0}$ should be understood as the average density. With these non-dimensional quantities, the governing equation can then be rewritten as

$$
\frac{\partial \hat{f}}{\partial \hat{t}}+\hat{c}_{k} \frac{\partial \hat{f}}{\partial \hat{x}_{k}}+\hat{g}_{k} \frac{\partial \hat{f}}{\partial \hat{c}_{k}}=-\frac{p_{0} L}{\mu_{0} \sqrt{R T_{0}}} \frac{\hat{p}}{\hat{\mu}}\left(\hat{f}-\hat{f}^{e q}\right)=-\frac{\hat{\rho} \hat{T}^{(1-\omega)}}{\mathcal{K}}\left(\hat{f}-\hat{f}^{S}\right),
$$

where $\mathcal{K}$ is defined as

$$
\mathcal{K}=\frac{\mu_{0} \sqrt{R T_{0}}}{p_{0} L}
$$

The relevant macroscopic quantities become

$$
\left[\begin{array}{c}
\hat{\rho} \\
\hat{\rho} \hat{u}_{i} \\
\hat{P}_{i j} \\
3 \hat{\rho} \hat{T} \\
2 \hat{q}_{i}
\end{array}\right]=\int \hat{f}\left[\begin{array}{c}
1 \\
\hat{C}_{i} \\
\hat{C}_{i} \hat{C}_{j} \\
\hat{C}_{i} \hat{C}_{i} \\
\hat{C}_{i} \hat{C}_{j} \hat{C}_{j}
\end{array}\right] d \hat{c} .
$$


The temperature-dependent viscosity can be expressed as $\mu / \mu_{0}=\left(T / T_{0}\right)^{\omega}$, where $\omega$ is related to the molecular interaction model, varing from 0.5 for hard-sphere molecular interactions to 1 for Maxwell molecules. The hat symbol will be omitted hereafter for clarity. A rescaled Knudsen number,

$$
K n=\sqrt{\frac{\pi}{2}} \mathcal{K},
$$

is used throughout this work.

\section{Numerical method}

\subsection{Equations of marginal distribution functions}

As the flows considered here are one-dimensional, we can introduce the following marginal velocity distribution functions, and the corresponding parts for the "equilibrium" distribution:

$$
\left[\begin{array}{c}
\varphi_{a} \\
\varphi_{b} \\
\varphi_{c} \\
\varphi_{d} \\
\varphi_{e} \\
\varphi_{f}
\end{array}\right]=\int_{-\infty}^{\infty} \int_{-\infty}^{\infty}\left[\begin{array}{c}
1 \\
c_{x} \\
c_{x}^{2} \\
c_{z}^{2} \\
c_{x}^{3} \\
c_{x} c_{z}^{2}
\end{array}\right] f d c_{x} d c_{z}
$$

$$
\left.\begin{array}{c}
{\left[\begin{array}{c}
\varphi_{a s} \\
\varphi_{b s} \\
\varphi_{c s} \\
\varphi_{d s} \\
\varphi_{e s} \\
\varphi_{f s}
\end{array}\right]=\int_{-\infty}^{\infty} \int_{-\infty}^{\infty}\left[\begin{array}{c}
1 \\
c_{x} \\
c_{x}^{2} \\
c_{z}^{2} \\
c_{x}^{3} \\
c_{x} c_{z}^{2}
\end{array}\right] f_{S} d c_{x} d c_{z}=\frac{\rho e^{-\frac{c_{y}^{2}}{2 T}}}{\sqrt{2 \pi T}} \times} \\
\frac{1}{5 \rho T^{3}}(\operatorname{Pr}-1)\left(-c_{y}^{3} q_{y} u_{x}-c_{y}^{2} q_{x} T+3 c_{y} q_{y} T u_{x}+q_{x} T^{2}\right)+u_{x} \\
1-\frac{1}{5 \rho T^{3}} c_{y} q_{y}(\operatorname{Pr}-1)\left(c_{y}^{2}-3 T\right) \\
\frac{1}{5 \rho T^{3}}(\operatorname{Pr}-1)\left(-c_{y}^{3} q_{y}\left(T+u_{x}^{2}\right)-2 c_{y}^{2} q_{x} T u_{x}+c_{y} q_{y} T\left(T+3 u_{x}^{2}\right)+2 q_{x} T^{2} u_{x}\right)+T+u_{x}^{2} \\
\frac{1}{5 \rho T^{2}} c_{y}(\operatorname{Pr}-1) q_{y}\left(T-c_{y}^{2}\right)+T \\
\frac{-1}{5 \rho T^{3}}(\operatorname{Pr}-1)\left(c_{y}^{3} q_{y} u_{x}\left(3 T+u_{x}^{2}\right)+3 c_{y}^{2} q_{x} T\left(T+u_{x}^{2}\right)-3 c_{y} q_{y} T u_{x}\left(T+u_{x}^{2}\right)+3 q_{x} T^{2}\left(T-u_{x}^{2}\right)\right)+u_{x}\left(3 T+u_{x}^{2}\right) \\
T u_{x}-\frac{1}{5 \rho T^{2}}(\operatorname{Pr}-1)\left(q_{x} T\left(c_{y}^{2}+T\right)+c_{y} q_{y} u_{x}\left(c_{y}^{2}-T\right)\right)
\end{array}\right] .
$$


With these marginal distribution functions, the macroscopic quantities in Eq. (2.4) can be calculated as

$$
\left[\begin{array}{c}
\rho \\
\rho u_{x} \\
3 \rho T \\
P_{x x} \\
P_{x y} \\
P_{y y} \\
P_{z z} \\
2 q_{x} \\
2 q_{y}
\end{array}\right]=\int_{-\infty}^{\infty}\left[\begin{array}{c}
\varphi_{a} \\
\varphi_{b} \\
\varphi_{c}+\varphi_{d}+c_{y}^{2} \varphi_{a} \\
\varphi_{c} \\
\varphi_{b} c_{y} \\
c_{y}^{2} \varphi_{a} \\
\varphi_{d} \\
-3 u_{x} \varphi_{c}+\varphi_{e}-u_{x} c_{y}^{2} \varphi_{a}+c_{y}^{2} \varphi_{b}-u_{x} \varphi_{d}+\varphi_{f} \\
c_{y}\left(-2 u_{x} \varphi_{b}+\varphi_{c}+c_{y}^{2} \varphi_{a}+\varphi_{d}\right)
\end{array}\right] d c_{y}+\left[\begin{array}{c}
0 \\
0 \\
-\rho u_{x}^{2} \\
-\rho u_{x}^{2} \\
0 \\
0 \\
0 \\
2 \rho u_{x}^{3} \\
0
\end{array}\right]
$$

It is worth noting that, for the unidirectional flows considered here, there are some additional relations, i.e. $u_{y}=u_{z}=0, P_{x z}=P_{y z}$ and $q_{z}=0$ [23]. Now let

$$
\phi=\left[\begin{array}{c}
\varphi_{a} \\
\varphi_{b} \\
\varphi_{c} \\
\varphi_{d} \\
\varphi_{e} \\
\varphi_{f}
\end{array}\right], \quad \phi_{e}=\left[\begin{array}{c}
\varphi_{a s} \\
\varphi_{b s} \\
\varphi_{c s} \\
\varphi_{d s} \\
\varphi_{e s} \\
\varphi_{f s}
\end{array}\right], S=g_{x}\left[\begin{array}{c}
0 \\
\varphi_{a} \\
2 \varphi_{b} \\
0 \\
3 \varphi_{c} \\
\varphi_{d}
\end{array}\right],
$$

then the governing equation for the six distribution functions can be written as

$$
\frac{\partial \phi}{\partial t}+c_{y} \frac{\partial \phi}{\partial y}=\frac{1}{\mathcal{K}} \rho T^{(1-\omega)}\left(\phi_{e}-\phi\right)+S .
$$

In particular, if the problem is steady, Eq. (3.3) can be further reduced to

$$
c_{y} \frac{\partial \phi}{\partial y}=\frac{1}{\mathcal{K}} \rho T^{(1-\omega)}\left(\phi_{e}-\phi\right)+S,
$$

where the time variable is eliminated. In these equations, the corresponding differential force terms have been transformed into the non-differential source term $S$ by utilizing integration by parts, see [24] for details.

\subsection{Scheme}

The flows are fully-developed, and we use the discrete velocity method to solve Eq. (3.4). We need to discretize in a two-dimensional space, i.e. one dimension in the physical space $y$, and the other dimension in the molecular velocity space $c_{y}$. For the molecular velocity space, Simpson's rule is chosen for $c_{y}$, and the grid points (say, $N_{c}$ points) are distributed uniformly. For the physical space, nonuniform grid points are employed with 
more points near the boundaries. To construct this kind of grids, we first obtain a distribution of grid points that become highly dense near the middle point of the channel by using

$$
y_{i}=\alpha \sinh \left[\frac{\sinh ^{-1}\left(\frac{1}{2 \alpha}\right)(2 i-N)}{N}\right]+\frac{1}{2}, \quad i=0 \ldots N,
$$

where $N$ is the total number of grid points and $\alpha$ is the parameter determining the nonuniformity. The grid system can then be made instead denser near the wall by utilizing symmetry and translation relations.

Regarding the numerical scheme, we employ a second-order upwind scheme except in the near-wall region where a first-order upwind scheme is used. Therefore, the evolution of $\phi$ can be written associated

$$
\phi_{i}=\frac{c_{y}\left(\eta_{i}^{2} \phi_{i-1}-\phi_{i-2}\right)+d y_{i} \eta_{i}\left(\eta_{i}-1\right)\left(w_{i} \phi_{e, i}+S_{i}\right)}{\left(\eta_{i}-1\right)\left(\eta_{i} c_{y}+c_{y}+d y_{i} \eta_{i} w_{i}\right)}, c_{y}>0, \quad i=2 \ldots N
$$

and

$$
\phi_{1}=\frac{c_{y} \phi_{0}+d y_{1} S_{1}+d y_{1} w_{1} \phi_{e, 1}}{c_{y}+d y w_{1}}, c_{y}>0
$$

where

$$
\begin{gathered}
w_{i}=\frac{\rho_{i} T_{i}^{1-\omega}}{\mathcal{K}}, \\
d y_{i}=y_{i}-y_{i-1}, \quad i=1 \ldots N,
\end{gathered}
$$

and

$$
\eta_{i}=\frac{d y_{i}+d y_{i-1}}{d y_{i}}, \quad i=2 \ldots N
$$

For simplicity, the rules for $c_{y}<0$ are omitted here; they can be easily obtained in a manner similar to the above. On the other hand, convergence tests show that the whole scheme can actually achieve a second-order accuracy even at the wall point although a first order scheme is used. This can be attributed to the usage of non-uniform grid. The accuracy near wall is effectively improved by the very small grid size.

\subsection{Boundary condition}

In the present simulations, diffuse reflection boundary conditions are employed. The top plate has a fixed temperature while the bottom plate has a specified heat flux. For convenience, the implementation details of both fixed-temperature and specified heat flux boundary will be discussed using the bottom wall as an example, which can then be straightforwardly applied to the top plate as required.

Supposing that the macroscopic properties at the wall, such as the density $\rho_{w}$, the velocity $\boldsymbol{u}_{w}$ and the temperature $T_{w}$ are known, the outgoing distribution function can be 


\begin{tabular}{llll}
\hline \hline$K n$ & $g_{x}$ or $U_{w}$ & $N$ & $N_{c}$ \\
\hline $0.05 \cdots 10$ & $U_{w}=0.2$ & 200 & 10000 \\
$0.05 \cdots 0.6$ and $0.8 \cdots 10$ & $U_{w}=1.5$ & 200 & 10000 \\
0.7 & $U_{w}=1.5$ & 400 & 10000 \\
$0.05 \cdots 3$ & $g_{x}=0.1$ and 1 & 200 & 10000 \\
5 & $g_{x}=1$ & 200 & 10000 \\
10 & $g_{x}=1$ & 200 & 20000 \\
5 & $g_{x}=0.1$ & 200 & 20000 \\
10 & $g_{x}=0.1$ & 200 & 80000 \\
\hline \hline
\end{tabular}

Table 1: Discretization systems for the simulated cases

written as follows,

$$
f\left(y=0, c_{y}>0\right)=\frac{\rho_{w}}{\left(2 \pi T_{w}\right)^{3 / 2}} \exp \left(-\frac{C_{w}^{2}}{2 T_{w}}\right),
$$

where $C_{w}$ is the peculiar molecular velocity at the wall, i.e. $\boldsymbol{c}-\boldsymbol{u}_{w}$. To implement the specific fixed-temperature and specified heat flux boundary conditons, we need to determine the corresponding macroscopic properties according to the related conservation laws. For simplicity, we first write down the incoming mass flux and heat flux as

$$
\begin{gathered}
\mathcal{M}_{\text {in }}=\int_{\mathcal{C}_{y}<0}\left|c_{y} f\left(y=0, c_{y}<0\right)\right| d c, \\
\mathcal{H}_{\text {in }}=\frac{1}{2} \int_{\mathcal{c}_{y}<0}\left|c_{y} C_{w}^{2} f\left(y=0, c_{y}<0\right)\right| d c .
\end{gathered}
$$

According to the princile of dissfuse reflection boundary conditons, the outgoing mass flux and heat flux can be easiy calcualted if $\rho_{w}$ and $T_{w}$ is first supposed to be known, i.e.,

$$
\mathcal{M}_{\text {out }}=\int_{\mathcal{C}_{y}>0}\left|c_{y} \frac{\rho_{w}}{\left(2 \pi T_{w}\right)^{3 / 2}} \exp \left(-\frac{C_{w}^{2}}{2 T_{w}}\right)\right| d c=\frac{\rho_{w} \sqrt{T_{w}}}{\sqrt{2 \pi}}
$$

and

$$
\mathcal{H}_{i n}=\frac{1}{2} \int_{\mathcal{C}_{y}<0}\left|c_{y} C_{w}^{2} \frac{\rho_{w}}{\left(2 \pi T_{w}\right)^{3 / 2}} \exp \left(-\frac{C_{w}^{2}}{2 T_{w}}\right)\right| d c=2 \sqrt{\frac{2}{\pi}} \rho_{w} T_{w}^{3 / 2} .
$$

If the wall temperature is fixed, we can use the mass conservation law to determine density, i.e. the incoming mass flux is equal to the outgoing mass flux. Through straightforward calculations, the density at wall is given as

$$
\rho_{w}=\sqrt{\frac{2 \pi}{T_{w}}} \mathcal{M}_{i n}
$$


For the wall with specified heat flux, we need to determine both density and temperature via mass conservation and the specified heat flux, i.e., we need to solve two equations as follows,

$$
\mathcal{M}_{\text {out }}-\mathcal{M}_{\text {in }}=0
$$

and

$$
\mathcal{H}_{\text {out }}-\mathcal{H}_{\text {out }}=q_{y w},
$$

where $q_{y w}$ denotes the specified heat flux at wall. By solving the two equations, the density and temperature can be written as

$$
\rho_{w}=\frac{2 \sqrt{2 \pi} \mathcal{M}_{i n}^{3 / 2}}{\sqrt{\mathcal{H}_{i n}+q_{y w}}}
$$

and

$$
T_{w}=\frac{\mathcal{H}_{i n}+q_{y w}}{4 \mathcal{M}_{i n}}
$$

Specifically, if $q_{y w}$ is set to be zero, the density and the temperature are

$$
\rho_{w}=\frac{2 \sqrt{2 \pi} \mathcal{M}_{i n}^{3 / 2}}{\sqrt{\mathcal{H}_{i n}}}
$$

and

$$
T_{w}=\frac{\mathcal{H}_{\text {in }}}{4 \mathcal{M}_{\text {in }}},
$$

Here, we will mainly study the effect of zero heat flux boundary condition and we suppose that the this boundary condition can closely resemble the adiabatic boundary condition. For the marginal distribution functions, the boundary Eq. (3.9) condition can be transformed to

$$
\left[\begin{array}{c}
\varphi_{a w} \\
\varphi_{b w} \\
\varphi_{c w} \\
\varphi_{d w} \\
\varphi_{e w} \\
\varphi_{f w}
\end{array}\right]=\int_{-\infty}^{\infty} \int_{-\infty}^{\infty}\left[\begin{array}{c}
1 \\
c_{x} \\
c_{x}^{2} \\
c_{z}^{2} \\
c_{x}^{3} \\
c_{x} c_{z}^{2}
\end{array}\right] f_{e q, w} d c_{x} d c_{z}=\frac{\rho_{w} \exp \left(-\frac{c_{y}^{2}}{2 T_{w}}\right)}{\sqrt{2 \pi T_{w}}}\left[\begin{array}{c}
1 \\
u_{w} \\
T_{w}+u_{w}^{2} \\
T_{w} \\
\left(3 T_{w}+u_{w}^{2}\right) u_{w} \\
T_{w} u_{w}
\end{array}\right]
$$

\section{Numerical results}

In the discrete velocity method, it is important to choose appropriate molecular velocities and spatial grids to ensure simualtion accuracy. We perform grid independent test in both physical space and velocity space, following the same procedure detailed in [24] where two types of errors are evaluated simultaneously to describe simulation accuracy. 

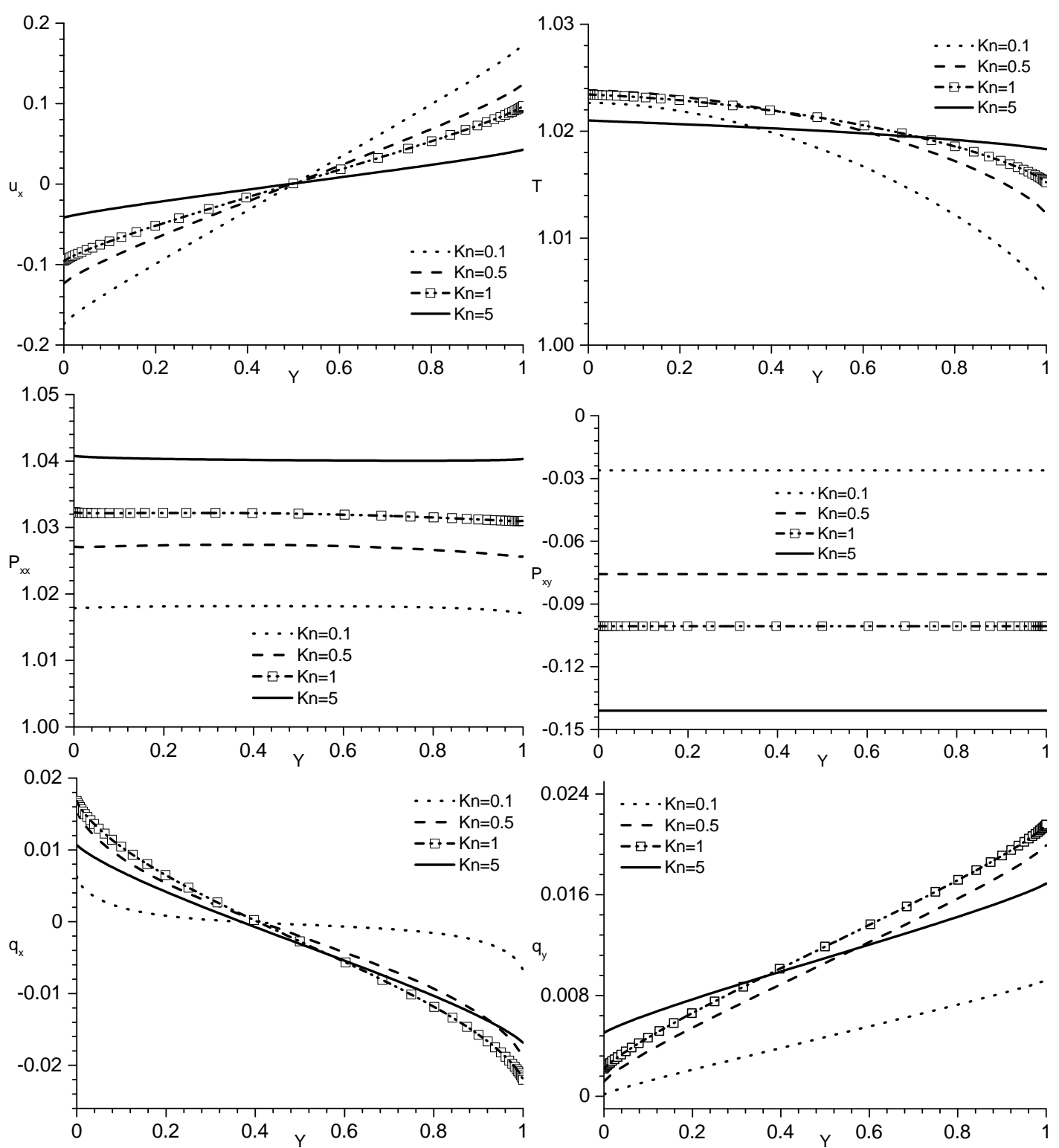

Figure 1: Profiles of macroscopic quantities for Couette flows with $U_{w}=0.2$. Adiabatic boundary at $Y=0$, fixed-temperature boundary at $Y=1$. 

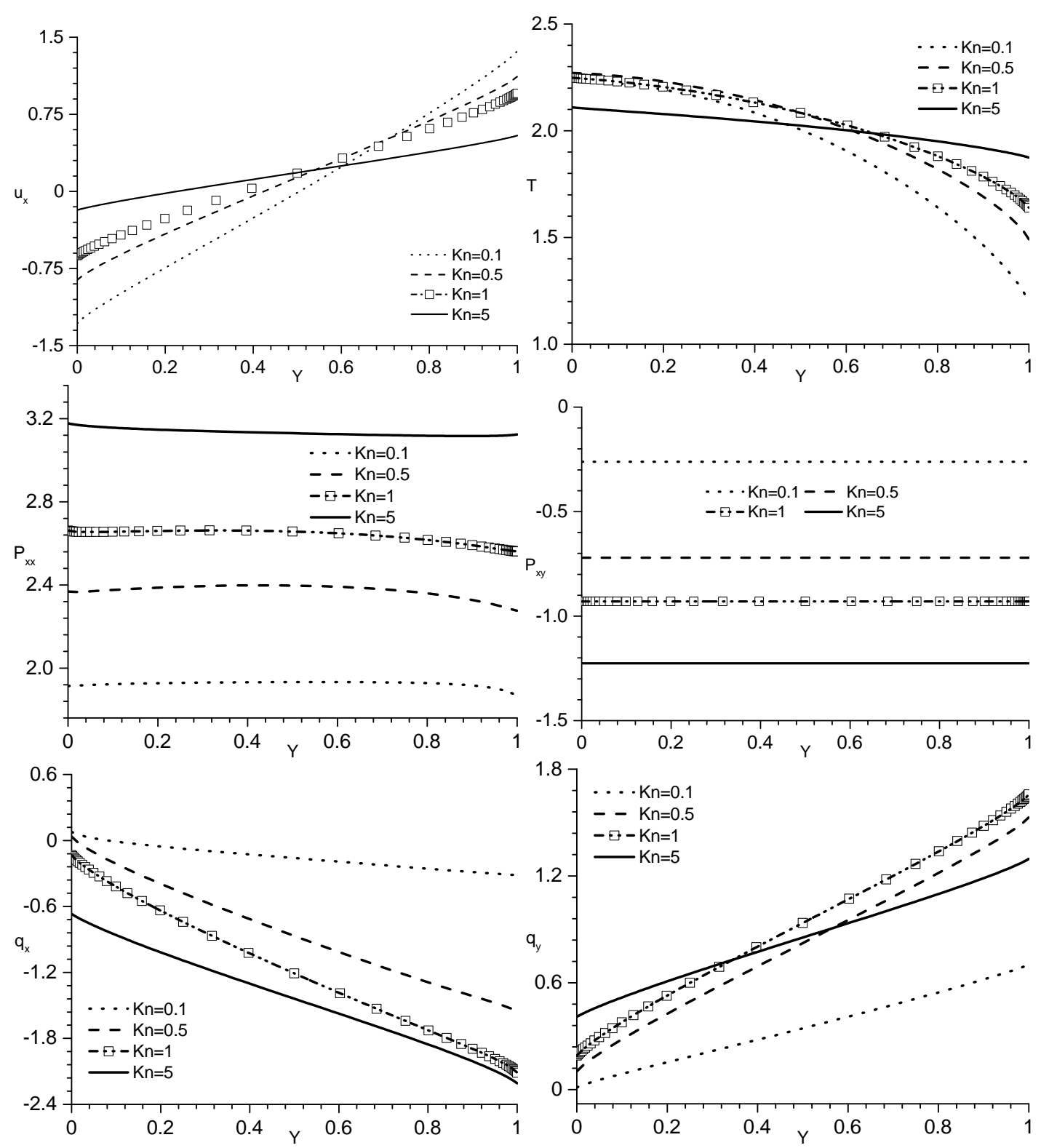

Figure 2: Profiles of macroscopic quantities for Couette flows with $U_{w}=1.5$. Adiabatic boundary at $Y=0$, fixed-temperature boundary at $Y=1$. 

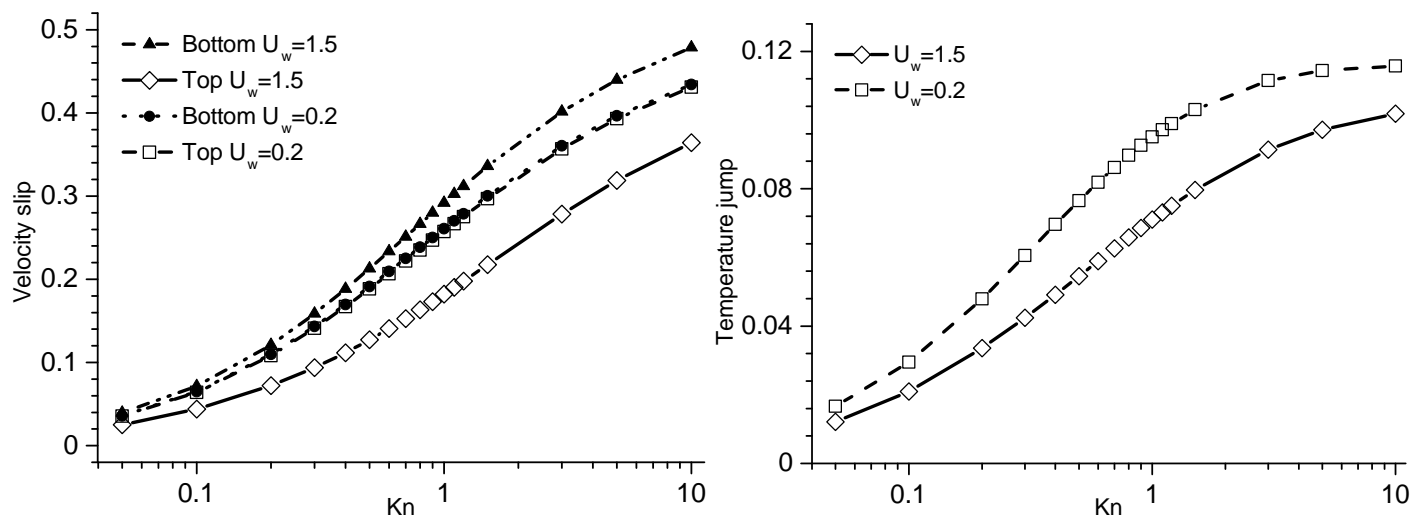

Figure 3: Velocity slip at the bottom (zero heat flux) and top (fixed-temperature) plates and temperature jump at the top plate, for Couette flows. The velocity slip is re-normalized by the velocity difference of the two moving plates, and the temperature jump is re-normalized by the square of the velocity difference.

Both types of error are evaluated at the points $[0,0.1,0.2, \cdots, 1]$ including the boundary points. If both types of errors for the temperature and velocity in the coarser system are less than $1 \%$ in comparison with the finer system, the coarser system is regarded as appropriate. According to these criteria, we determine an appropriately discretized system for each simulated case, which are listed in Table 11. In terms of the truncation of molecular velocity space, we choose the range $[-20,20]$, which produces nearly identical results for the force-driven flow with $K n=0.05$ and $g_{x}=1$ with the range $[-30,30]$.

Typical results for Couette flows are presented in Figs. 1-3. In Figs. 1 and 2 it is found that the viscous heating effect can induce a larger temperature rise at the bottom wall. For both $U_{w}=0.2$ and $U_{w}=1.5$ the temperature keeps rising at the top wall with the increasing Knudsen number in the presented region. However, at the bottom wall, it first increases then decreases. For the velocity, same trends are kept for both walls.

In Fig. 3 it is seen that the effect of the zero heat flux boundary on the velocity profile is significant for the high-speed case as we can observe significantly different velocity slips at the bottom and top plates. Specifically, the velocity slip at the bottom (zero heat flux) plate is larger. Interestingly, for the low-speed case, the effect on velocity slip is small. At the top plate, we observe that the temperature jump effect is relatively stronger for the lower speed case, in comparison to the higher speed case.

Regarding the heat flux, it is of interest to observe that there can be non-zero spanwise heat flux $q_{y}$ at the bottom wall for the larger Knudsen numbers, although a zero heat flux is prescribed. This is certainly due to the non-continuum effect and may be understood as a kind of heat flux jump. For the stream-wise component, we ale observe non-zero values which can not be captured by the NSF equations.

For the shear stress $P_{x x}$ and $P_{x y}$, they show similar behaviors for both of the higherspeed and lower-speed cases. Again, the trace-free part of shear stress $P_{x x}-T$ is apparently not zero, which can be be captured by the NSF equations.

The results for force-driven Poiseuille flows are shown in Figs. $4-8$. Similar to the 

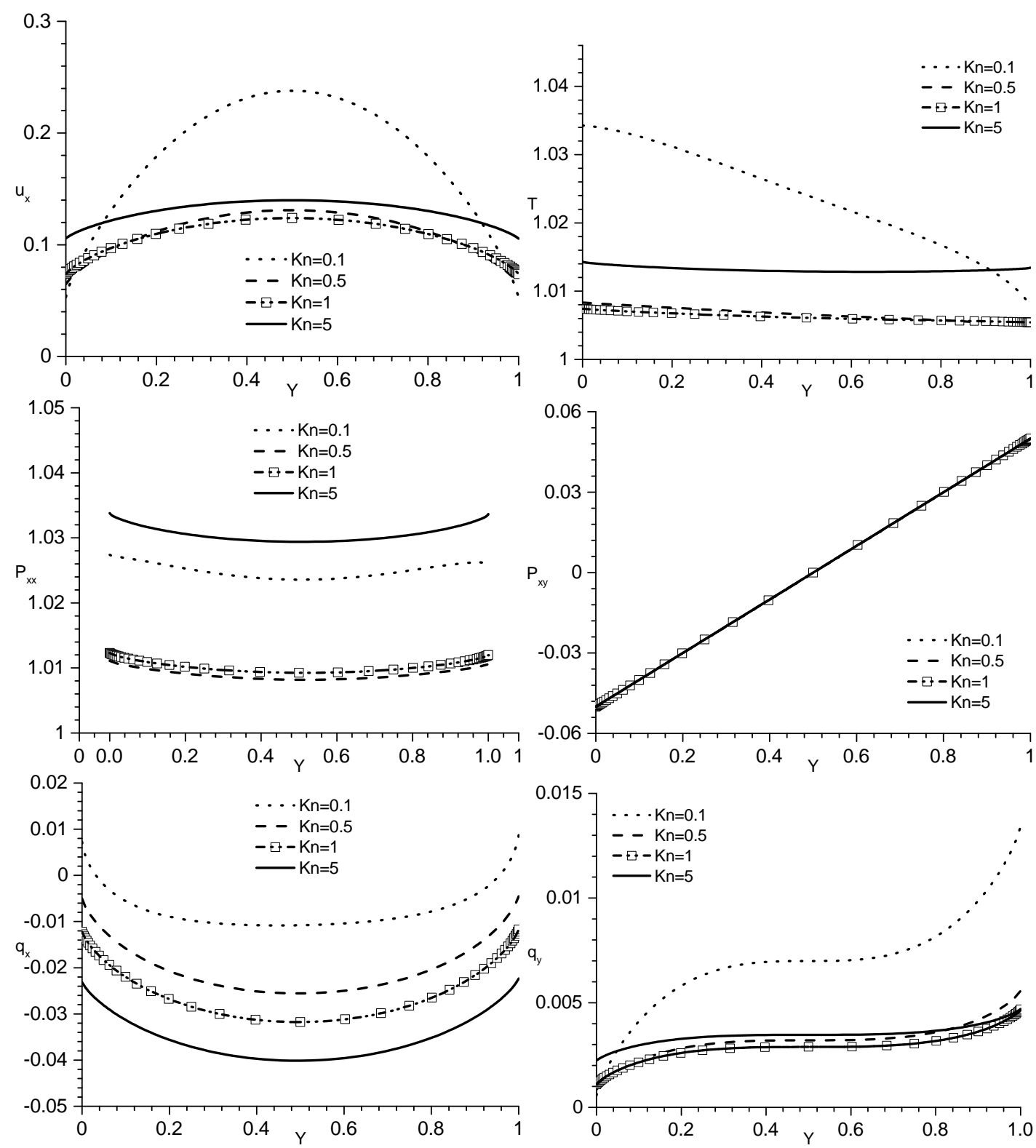

Figure 4: Profiles of macroscopic quantities for force-driven Poiseuille type flows with $g_{x}=0.1$. Adiabatic boundary at $Y=0$, fixed-temperature boundary at $Y=1$. 


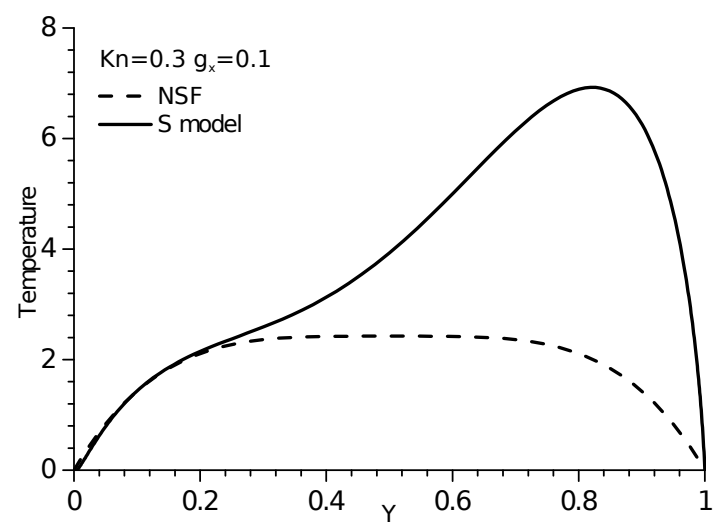

Figure 5: Comparison of temperature profiles predicted by the $\mathrm{S}$ model and the NSF equation at $K n=0.3$ and $g_{x}=0.1$. The temperature profiles as shown are deducted by data belonging to lines connecting points at the top and bottom plates and then enlarged by 10000 times respectively. The velocity slip boundary condition is used for both two walls. Whie the temperature jump boundary condition is used at the top wall and the zero heat flux (temperature gradient) boundary condition is used at the bottom wall.

Couette flow cases, the zero heat flux boundary induces significantly different velocity slips at the bottom and top plates for the case of $g_{x}=1$. Different from the Couette flow, the velocity slip at the bottom wall becomes smaller as the external force increases. Again there is nearly no difference in velocity slip at the two walls for the small force cases and the temperature jump effect is relatively stronger than the the large force case.

It is interesting to note that, similar to the case of two fixed-temperature plates, we can find that the temperature profiles are qualitatively different from the NSF prediction for a range of Knudsen numbers. For instance, as shown in Fig. 5, we can see bimodal-like temperature distribution although the two tops are unsymmetrical here. This is qualitatively different from the NSF prediction. Again the temperature near the zero heat flux wall is always the highest.

Similar to the Couette flow, heat flux jump is observed at the bottom wall. Also, there are non-zero stream-wise heat flux component and non zero trace-free shear stress $P_{x x}-T$.

In Fig. 8, we can see that, similar to cases with fixed temperature boundary at two walls, the Knudsen minimum in the mass flow rate occurs at around $K n=1$. Since the viscous heating effect consumes more input work in the larger force case, the mass flow rates are generally larger for the smaller force case.

\section{Concluding remarks}

To summarize, we have studied rarefied Couette and Poiseuille-type flows confined between two parallel plates with specified heat flux (mainly zero heat flux) and fixedtemperature walls. By numerically solving the $S$ model, we have seen that the zero heat flux wall has a significant impact on the flowfield. Overall the profiles of macro- 

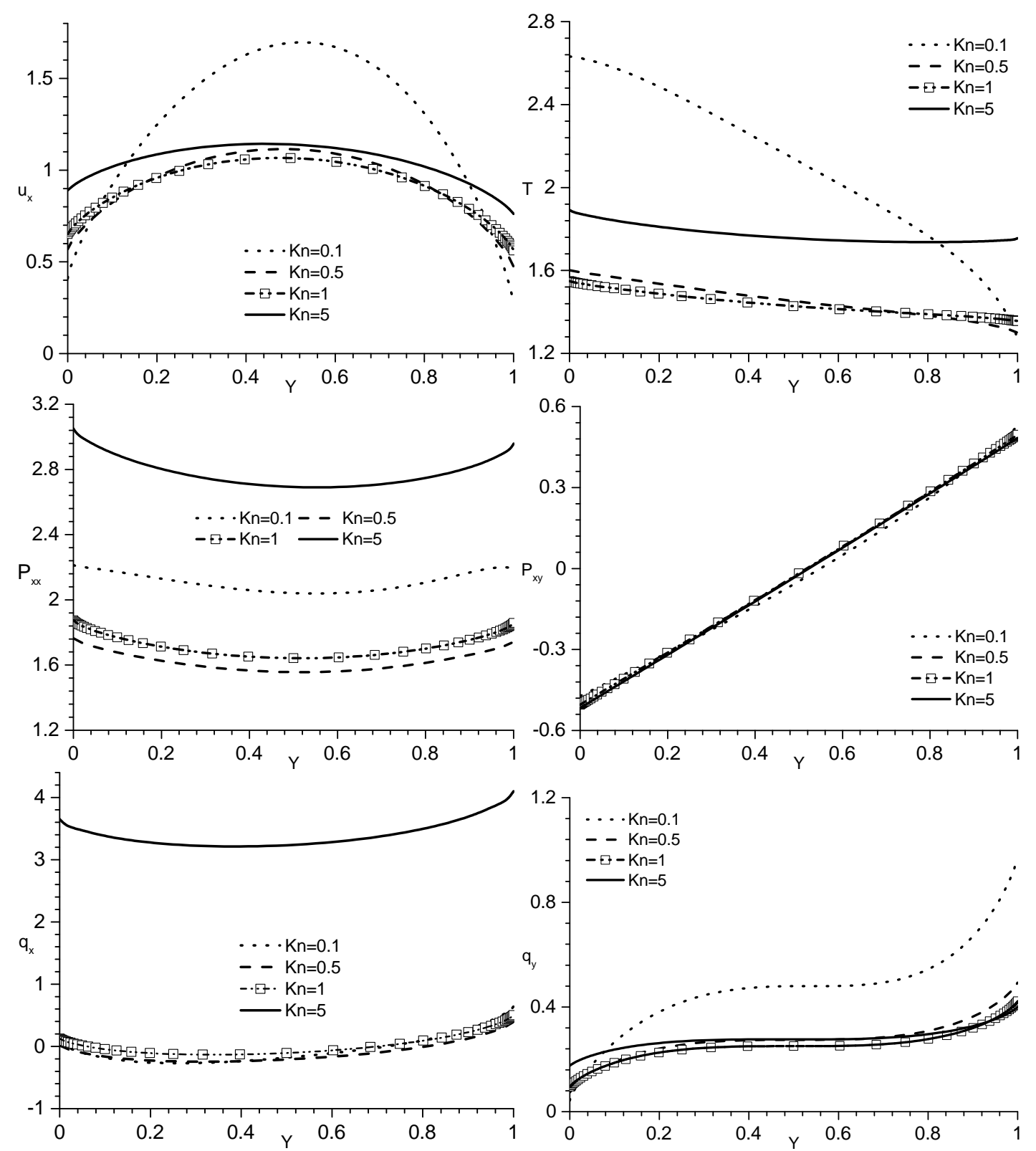

Figure 6: Profiles of macroscopic quantities for force-driven Poiseuille type flows with $g_{x}=1$. Adiabatic boundary at $Y=0$, fixed-temperature boundary at $Y=1$. 

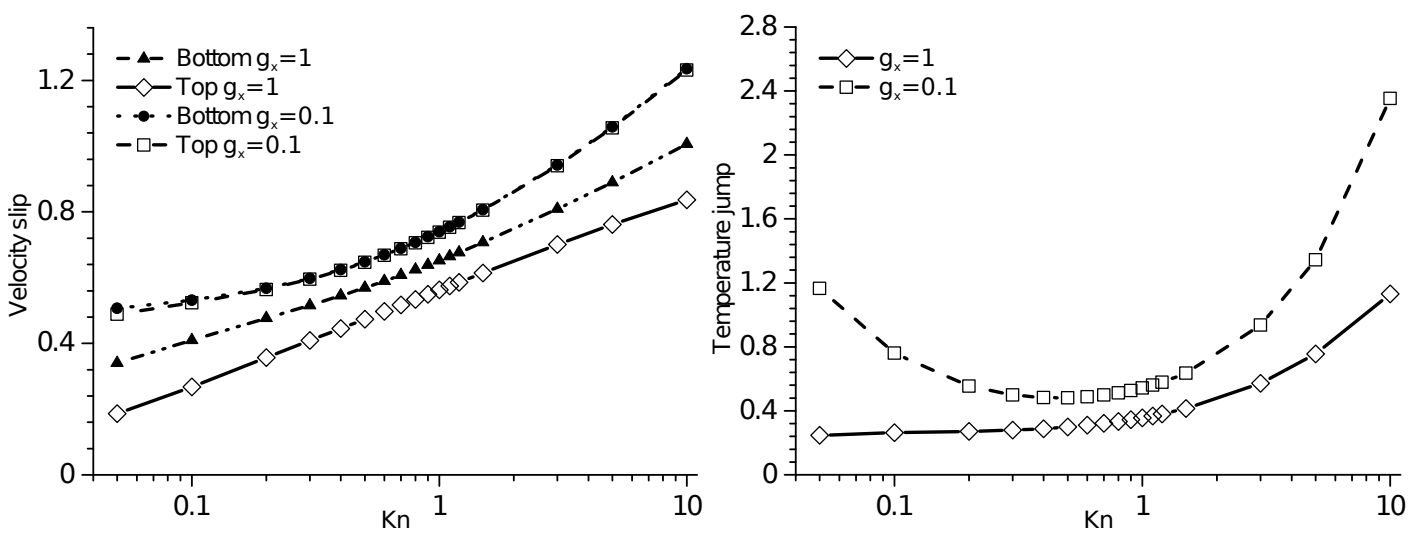

Figure 7: Velocity slip at the bottom (zero heat flux)and top (fixed-temperature) plates and temperature jump at the top plate, for force-driven Poiseuille type flows. The velocity slip is re-normalized by the force magnitude, and the temperature jump is re-normalized by the square of the force magnitude.
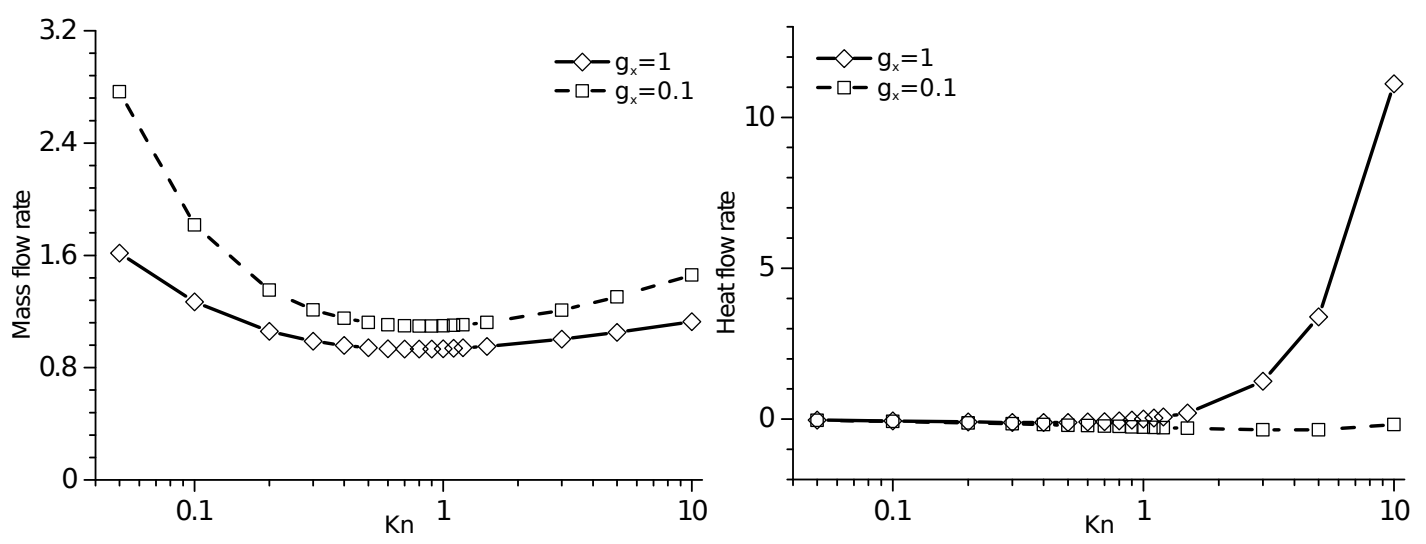

Figure 8: Mass and heat flow rate for force-driven Poiseuille type flows of different $K n$. Both fluid quantities are re-normalized by the force magnitude.

scopic fluid quantities can become unsymmetrical. Specifically, the temperature rise at the zero heat flux wall are higher than that at the fixed-temperature plate. When the viscous heating effect is strong (e.g. $U_{w}=1.5$, or $g_{x}=1$ ), the velocity slips at the two walls become significantly different. A interesting spans-wise heat flux jump phenomenon can be observed at the zero heat flux wall. For the Poiseuille-type flows, the temperature profiles are qualitatively different from hydrodynamic predictions, i.e. a bimodal-like distribution is found for a range of Knudsen numbers. Hence the Poiseuille-type flow appears to be a good benchmark problem for testing the capability of extended hydrodynamic models to capture the zero heat flux wall effect. 


\section{Acknowledgments}

This work was financially supported by the Engineering and Physical Sciences Research Council of the UK under Grant Nos. EP/I036117/1 and EP/I011927/1. Yonghao Zhang would also like to thank the Royal Academy of Engineering and the Leverhulme Trust for the award of a RAEng/Leverhulme Senior Research Fellowship.

\section{References}

[1] M. S. Ivanov and S. F. Gimelshein. Computational hypersonic rarefied flows. Annu. Rev. Fluid Mech., 30(1) (1998), 469-505.

[2] Chih-Ming Ho and Yu-Chong Tai. Micro-electro-mechanical-systems (MEMS) and fluid flows. Annu. Rev. Fluid Mech., 30(1) (1998), 579-612.

[3] G. A. Bird. Monte Carlo simulation of gas flows. Annu. Rev. Fluid Mech., 10(1) (1978), 11-31.

[4] S. M. Yen. Numerical solution of the nonlinear Boltzmann equation for nonequilibrium gas flow problems. Annu. Rev. Fluid Mech., 16(1) (1984), 67-97.

[5] Gregg A. Radtke, Nicolas G. Hadjiconstantinou, and Wolfgang Wagner. Low-noise Monte Carlo simulation of the variable hard sphere gas. Phys. Fluids, 23(3) (2011), 030606.

[6] H. Struchtrup. Derivation of 13 moment equations for rarefied gas flow to second order accuracy for arbitrary interaction potentials. Multiscale Model.Simul., 3(1) (2005), 221-243.

[7] Xiaojun $\mathrm{Gu}$ and David R. Emerson. A high-order moment approach for capturing nonequilibrium phenomena in the transition regime. J. Fluid Mech., 636(-1) (2009), 177-216.

[8] Jianping Meng, Yonghao Zhang, Nicolas G. Hadjiconstantinou, Gregg A. Radtke, and Xiaowen Shan. Lattice ellipsoidal statistical BGK model for thermal non-equilibrium flows. J. Fluid Mech., 718 (2013), 347-370.

[9] H. Akhlaghi, E. Roohi and S. Stefanov, A new iterative wall heat flux specifying technique in DSMC for heating/cooling simulations of MEMS/NEMS, Int. J. Therm. Sci., 59 (2012), 111-125.

[10] Q. W. Wang, C. L. Zhao, M. Zheng and N. Y. E. Wu, Numerical investigation of rarefied diatomic gas flow and heat transfer in microchannel using dsmc with heat flux specified boundary conditiond part I: Numerical method and validation, Numerical Heat Transfer Part $B, 53$ (2008), 160-173.

[11] T. Klinc and I. Kušer Slip coefficients for general gas-surface interaction, Phys. Fluids, 15 (1972), 1018-1022

[12] A. A. Alexeenko, D.A. Levin, S. F. Gimelshein, R. J. Collins and G.N.Markelov. Numerical simulation of high-temperature Gas flows in a millimeter-Scale thruster , J. Therm. Heat Trans., 16 (2002), 10-16.

[13] G. N. Markelov, A. N. Kudryavtsev and M. S. Ivanov, Rarefaction effects on separation of hypersonic laminar flows, AIP Conference Proceedings, 585 (2001), 707.

[14] G. N. Markelov and M. S. Ivanov, Numerical study of 2D/3D micronozzle flows, AIP Conference Proceedings, 585 (2001), 539.

[15] N Sengil and F. O. Edis. Implementation of parallel DSMC method to adiabatic piston problem. In Parallel Computational Fluid Dynamics 2007, volume 67 of Lecture Notes in Computational Science and Engineering, pages 75-82. Springer Berlin Heidelberg, 2009. 
[16] Alireza Mohammadzadeh, Ehsan Roohi, Hamid Niazmand, and Stefan Stefanov. Dsmc solution for the adiabatic and isothermal micro/nano lid-driven cavity. In Proceedings of the 3rd GASMEMS Workshop - Bertinoro, 2011.

[17] Rakesh Kumar, Evgeny Titov, and Deborah Levin. Comparison of statistical BGK and DSMC methods with theoretical solutions for two classical fluid flow problems. In Fluid Dynamics and Co-located Conferences, American Institute of Aeronautics and Astronautics, 2009.

[18] Jonathan M. Burt and Iain D. Boyd. Evaluation of a particle method for the ellipsoidal statistical Bhatnagar-Gross-Krook equation. In $44^{\text {th }}$ AIAA Aerospace Sciences Meeting and Exhibit, 2006.

[19] E. M. Shakhov. Generalization of the Krook kinetic relaxation equation. Fluid Dyn., 3(5) (1968), 95-96.

[20] E. M. Shakhov. Approximate kinetic equations in rarefied gas theory. Fluid Dyn, 3(1) (1968), 112-115.

[21] Tadeusz Platkowski and Reinhard Illner. Discrete velocity models of the Boltzmann equation: A survey on the mathematical aspects of the theory. SIAM Review, 30(2) (1988), 213-255.

[22] Yoshio Sone, Kinetic theory and fluid Dynamics, BIRKHÄUSER 2002.

[23] Kazuo Aoki, Shigeru Takata, and Toshiyuki Nakanishi. Poiseuille-type flow of a rarefied gas between two parallel plates driven by a uniform external force. Phys. Rev. E, 65 (2002), 026315.

[24] Jianping Meng, Lei Wu, Jason M. Reese, and Yonghao Zhang. Assessment of the ellipsoidalstatistical Bhatnagar-Gross-Krook model for force-driven Poiseuille flows. J. Comput. Phys., 251(0)(2013), 383-395. 